\title{
Blockade of Nogo Receptor Ligands Promotes Functional Regeneration of Sensory Axons after Dorsal Root Crush
}

\author{
Pamela A. Harvey, ${ }^{1}$ Daniel H. S. Lee, ${ }^{2}$ Fang Qian, ${ }^{2}$ Paul H. Weinreb, ${ }^{2}$ and Eric Frank ${ }^{1}$ \\ ${ }^{1}$ Department of Physiology, Tufts University School of Medicine, Boston, Massachusetts 02111, and ${ }^{2}$ Biogen Idec, Inc., Cambridge, Massachusetts 02142
}

A major impediment for regeneration of axons within the CNS is the presence of multiple inhibitory factors associated with myelin. Three of these factors bind to the Nogo receptor, $\mathrm{NgR}$, which is expressed on axons. Administration of exogenous blockers of NgR or NgR ligands promotes the regeneration of descending axonal projections after spinal cord hemisection. A more detailed analysis of CNS regeneration can be made by examining the growth of specific classes of sensory axons into the spinal cord after dorsal root crush injury. In this study, we assessed whether administration of a soluble peptide fragment of the $\mathrm{NgR}(\mathrm{sNgR})$ that binds to and blocks all three $\mathrm{NgR}$ ligands can promote regeneration after brachial dorsal root crush in adult rats. Intraventricular infusion of $\mathrm{sNgR}$ for 1 month results in extensive regrowth of myelinated sensory axons into the white and gray matter of the dorsal spinal cord, but unmyelinated sensory afferents do not regenerate. In concert with the anatomical growth of sensory axons into the cord, there is a gradual restoration of synaptic function in the denervated region, as revealed by extracellular microelectrode recordings from the spinal gray matter in response to stimulation of peripheral nerves. These positive synaptic responses are correlated with substantial improvements in use of the forelimb, as assessed by paw preference, paw withdrawal to tactile stimuli and the ability to grasp. These results suggest that sNgR may be a potential therapy for restoring sensory function after injuries to sensory roots.

\section{Introduction}

A major goal in the search for therapies for spinal cord injuries (SCIs) is to develop tools for promoting both the regeneration of damaged axons and the restoration of synaptic contacts with their appropriate targets. Neurons in the CNS normally do not regenerate after damage, in part because of the presence of factors that retard axonal growth in the mature brain. Several different proteins expressed on mature oligodendrocytes cause axonal growth cones to collapse and thus arrest further growth (Fawcett et al., 1989; Yiu and He, 2006). In addition, neurotrophic factors and growth-associated proteins, which are expressed in injured peripheral nerves, are often absent in the adult CNS. Axons may also be confronted by a proteoglycan-rich glial scar at the lesion site, which forms a physical and molecular barrier to regrowth (Goldberg and Barres, 2000; Schwab, 2004).

To date, three proteins in CNS myelin have been identified as inhibitors of axon growth: myelin-associated glycoprotein (MAG), oligodendrocyte glycoprotein (OMgp), and Nogo. All three bind the same receptor, the Nogo-66 receptor (NgR1), which is expressed by many neurons in the CNS (Hunt, 2002). Antibody blockade or genetic deletion of NgR1 does not com-

\footnotetext{
Received Dec. 10, 2008; revised March 13, 2009; accepted April 13, 2009.

This work was supported by contracts from Biogen Idec, Inc., a grant from the Paralyzed Veterans Association Research Foundation (\#2457), and a gift from the Murray Winston Foundation. We thank Peter Cariani, Jason Cain, and Lucas Marzec, who were instrumental in pilot experiments in this study. Dr. Sonal Jhaveri made helpful contributions to this manuscript.

Correspondence should be addressed to Eric Frank, Department of Physiology, Tufts University School of Medicine, 136 Harrison Avenue, Boston, MA 02111. E-mail: eric.frank@tufts.edu.

D. H. S. Lee's present address: GlaxoSmithKline Research and Development Centre, Shanghai 201203, China. D0I:10.1523/JNEUROSC1.5885-08.2009

Copyright $\odot 2009$ Society for Neuroscience $\quad$ 0270-6474/09/296285-11\$15.00/0
}

pletely eliminate the inhibitory influence of these proteins, however (Kim et al., 2004; Zheng et al., 2005; Chivatakarn et al., 2007), suggesting that other receptors also participate in mediating their inhibitory effects. For example, a second Nogo receptor, $\mathrm{NgR} 2$, contributes to the inhibitory effect of MAG on neurite growth (Venkatesh et al., 2005), and, recently, the paired immunoglobulin-like receptor B (PirB) has been shown to bind MAG, Nogo, and OMpg and to mediate a major part of their inhibitory influence (Atwal et al., 2008). A peptide that binds to all three proteins might block their interactions with multiple receptors and, therefore, be more effective in blocking inhibition. One candidate peptide is the ectodomain of $\mathrm{NgR} 1$, which includes the entire binding region for Nogo66 (Fournier et al., 2002), coupled to an antibody fragment (Li et al., 2004) termed soluble $\mathrm{NgR}(\mathrm{sNgR})$. Treatment with $\mathrm{sNgR}$ promotes sprouting of several classes of spinal axons after both dorsal spinal cord hemisection (Ji et al., 2005; Li et al., 2005; Wang et al., 2006) and multiple dorsal root rhizotomy (MacDermid et al., 2004), but possible effects of $\mathrm{sNgR}$ on regeneration of lesioned axons have not been reported.

A persistent challenge to the field has been to identify unambiguously regenerating axons from axons that are spared by a lesion and have sprouted in response to the damage. The dorsal root crush paradigm used in this study provides an excellent model for overcoming these difficulties in the study of myelinassociated inhibition. When sensory axons are lesioned by dorsal root crush, the axons regenerate within the root up to the dorsal root entry zone (DREZ). Axonal growth stops at this site, however, and entry into the CNS fails (Ramon y Cajal, 1928; Carlstedt, 1997). In contrast to contusion or transection models of SCI, crushing the dorsal root interrupts virtually all sensory ax- 
ons. Moreover, the projection patterns of sensory axons into the spinal cord and the physiological responses of spinal neurons to stimulation of these axons are well characterized. The extent of the central projections of regenerated sensory axons can be specifically assessed by applying neurotracers to their peripheral processes. Finally, the location of these processes makes them accessible to electrical stimulation, thereby permitting detailed electrophysiological studies of how regenerating axons reconstitute functional circuits within the spinal cord.

In this study, we tested whether $\mathrm{sNgR}$ promotes regeneration of sensory axons into the spinal cord after dorsal root crush in adult rats. We show that $\mathrm{sNgR}$ treatment results in extensive regrowth of sensory axons into the white and gray matter of the dorsal spinal cord. Electrophysiological recordings demonstrate that these regenerating axons re-establish functional synaptic connections with spinal neurons. Finally, sNgR-treated rats show substantial improvements in the use of their affected limbs.

\section{Materials and Methods}

All research was approved by the Institutional Animal Care and Use Committee at Tufts University School of Medicine and conformed to National Institutes of Health guidelines. Fifty-seven rats were used (see below); of these, eight animals were excluded from the study because of autotomy of forelimb digits or skin ipsilateral to injury within $14 \mathrm{~d}$ of crush lesion. The incidence of autotomy did not correlate with the treatment administered. One animal was excluded after determining that the infusion port was improperly placed in the brain.

We developed criteria to identify animals with possible incomplete sensory root crushes based on behavioral observations and electrophysiological recordings. Rapid recovery of responses may suggest that some axons survived the crush. Animals that regained the ability to grasp (normally not seen until the third postoperative week in sNgR-treated animals) within the first two postoperative weeks were, therefore, excluded. Synaptic responses on the regenerated side of $\mathrm{sNgR}$-treated animals typically had longer latencies and slower falling phases than responses observed on the uninjured side. When latencies or falling phases were not delayed or slowed, the animal was also excluded. Based on these criteria, three animals were excluded from this study; two sNgR-treated animals grasped during the second postoperative week, and one sNgR-treated animal had near-normal synaptic response latency. No control animals were excluded based on these criteria.

\section{Dorsal root lesions}

All surgeries were performed aseptically. Sprague Dawley rats (200-250 g; Charles River Laboratories) were anesthetized with 2-3\% isoflurane; the level of anesthesia was monitored by checking response to foot pinch. Rectal temperature, blood oxygen saturation, and cardiac rate were also monitored. The cervical spinal cord and $\sim 2 \mathrm{~mm}$ of the dorsal roots were exposed via a partial dorsal laminectomy. The five roots that innervate the forelimb via the radial, median, and ulnar nerves (C5-T1) were identified using the T2 spinal process as a landmark. Dorsal roots supplying these nerves were crushed unilaterally, as illustrated in Figure $1 \mathrm{~A}$, between the dorsal root ganglia and DREZ using fine forceps (Dumostar \#7). The dura was pierced with one tine, and the root was crushed against the dura for $3 \mathrm{~s}$. Crushes were repeated 2-3 times per root in a location close to the original crush (Ramer et al., 2000; Romero et al., 2001; Wang et al., 2008). Overlying muscles were sutured, and the skin incision was closed with steel autoclips or by suturing.

In most rats, we crushed five roots (C5-T1) to ensure that all sensory inputs from the forelimb were interrupted. In $10 \mathrm{sNgR}$-treated rats, however, only the $\mathrm{C} 6-\mathrm{C} 8$ roots were crushed. The results from these rats are included in the data presented in Figure $5 B$ (10 of 17 rats with survival times of 5 or 6 weeks) but not in any of the anatomical or behavioral assessments of regeneration. For all rats in this group, electrophysiological tests in the terminal experiment provided evidence that all the responses evoked by peripheral nerve stimulation were mediated by sensory axons in the three crushed roots, because these responses were completely abolished by cutting these roots.
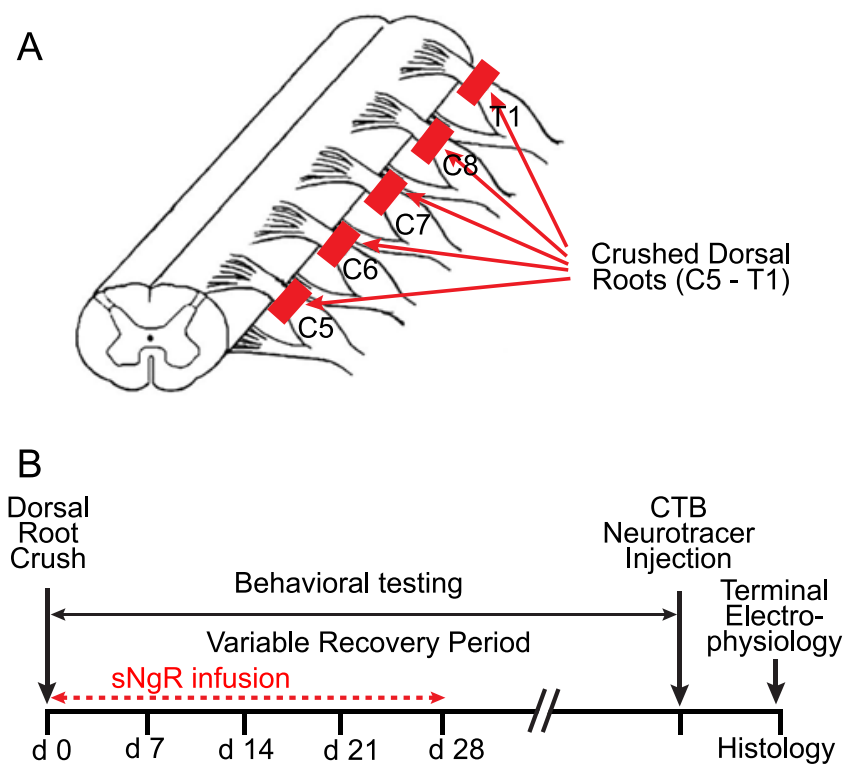

Figure 1. A, Schematic diagram of experimental preparation. All cervical dorsal roots that supply the brachial plexus, from (5-T1, were crushed, as described in Materials and Methods. $\boldsymbol{B}$, Experimental time course. Dorsal roots were crushed in an initial surgery, and infusion of $s N g R$ was begun immediately into the lateral ventricle via osmotic minipump and was continued for $28 \mathrm{~d}$. Behavioral testing was performed once or twice each week for the duration of the recovery period. One week before electrophysiological recording, peripheral nerves were injected with CTB. After the recording session, rats were perfused and the spinal cords processed for histology.

\section{sNgR treatment}

Samples of sNgR were prepared and coded before use by Biogen Idec, Inc. The form of sNgR used for this study, $\mathrm{AA}-\mathrm{rNgR}(310)-\mathrm{rFc}$, is an engineered variant of the $\mathrm{NgR}-$ ecto-Fc fusion protein reported previously (Li et al., 2004). This protein comprises a 310 aa fragment of rat $\mathrm{NgR} 1$ fused to a rat IgG1 Fc fragment, in which Cys266 and Cys309 were replaced with alanine residues to eliminate heterogenous disulfide bonds. The construct was expressed in Chinese hamster ovary cells, protein was purified, and binding to Nogo66, OMgp, and MAG was verified (Li et al., 2004). This modified protein inhibits the Nogo66-NgR interaction and promotes neurite outgrowth in vitro with similar potency as the unmodified NgR-ecto-Fc (P. Weinreb, F. Qian, M.-Y. Jung, and D. Lee, unpublished observations). Samples of $s \mathrm{NgR}$ or vehicle were continuously infused ( $1 \mathrm{mg}$ over $28 \mathrm{~d}$ ) into the lateral cerebral ventricle by means of an osmotic minipump (ALZET; Model 2004) connected to a brain infusion port by a flexible plastic cannula (ALZET; Brain Infusion Kit II). In separate tests, the stability of $\mathrm{sNgR}$ at $37^{\circ} \mathrm{C}$ was evaluated for up to 1 month. Moderate increases in the amount of soluble aggregates were observed over time (an increase of $\sim 5 \%$ per week, as determined using size-exclusion chromatography), but the samples maintained stable activity in ligand-binding and neurite growth inhibition assays (data not shown). Based on an earlier study, $\mathrm{sNgR}$ penetrated into the spinal cord for $\sim 300 \mu \mathrm{m}$, including the dorsolateral funiculus and dorsal and ventral horns (MacDermid et al., 2004). Anatomical, behavioral, and electrophysiological measurements and analyses were done blind, before decoding samples. A timeline for all experimental procedures is shown in Figure $1 B$.

In four rats, the human variant of $\mathrm{sNgR}$ was used instead of the rat variant. This molecule, $\mathrm{AA}-\mathrm{hNgR} 1(310)-\mathrm{hFc}$, contained the homologous human 1-310 sequence fused to a human IgG1 Fc domain, with the analogous pair of Cys to Ala substitutions as introduced into the rat $\mathrm{sNgR}$. The human and rat molecules showed comparable in vitro properties, including binding to Nogo-66 and promotion of neurite outgrowth (Weinreb et al., unpublished observations). These four rats were included in the behavioral analyses, and two of these rats were also tested electrophysiologically at 4 weeks after root crush. The results obtained 
with the human variant were indistinguishable from those obtained with the rat variant of $s \mathrm{NgR}$ and were included in the overall data set used for analysis.

\section{Behavioral analyses}

Grasping reflex. Recovery of the ability to grasp was evaluated at 3-5 d intervals throughout the treatment period. Animals were suspended by the tail above a horizontal bar $(7.5 \mathrm{~cm}$ long and $\sim 3 \mathrm{~mm}$ in diameter $)$ located $\sim 2 \mathrm{~cm}$ above the cage top, slowly lowered until both paws came in contact with the bar for less than one second, and lifted again. If the animal grasped the bar strongly, and both forepaws released nearly simultaneously when pulled away, a score of 2 was assigned. If the animal grasped the bar with both paws but released with the paw ipsilateral to dorsal root crush before releasing with the unaffected one, a score of 1 was assigned. A score of 0 was assigned if the animal could not position the paw on the bar or did not exhibit spontaneous grasping. For each test, three trials were performed. Often, especially at early time points, the behavior was inconsistent, in which case the best response was recorded.

Limb-use asymmetry test. Also referred to as a paw preference test, this behavioral assay was used to evaluate the recovery of general use of the compromised limb (Schallert et al., 2000). Animals were placed in a glass cylinder (30 cm high, $20 \mathrm{~cm}$ diameter); exploratory behavior was recorded for $4.5 \mathrm{~min}$ with a digital camera and, at a later time, was evaluated in slow motion. We recorded the number of times the animal placed weight on each paw against the cylinder wall (i.e., the entire paw was in contact with the glass). Light paw or digit touches did not qualify as weight-bearing placements. The placement score was calculated as the ratio of affected to unaffected paw placements; a score of 1.0 indicates equal use of both paws.

Touch sensitivity. To assess sensitivity of the affected forepaw, we used the von Frey Hair Test. Animals were placed on a suspended wire grate. Testing began after $\sim 10 \mathrm{~min}$, once the animal was resting quietly. Semmes-Weinstein monofilaments calibrated to deliver forces between 4 and $180 \mathrm{~g}$, which is the average sensitivity range for uninjured rats, were used to determine the level of force that caused the animal to lift its forepaw on the compromised side. The 5.18 monofilament $(15 \mathrm{~g})$ is reported to be the midpoint of sensitivity for an uninjured paw (Lindsey et al., 2000). Monofilaments were touched to the forepaw surface for $2 \mathrm{~s}$ with sufficient force to bend the monofilament. Each touch constituted one trial. Withdrawal of the paw was scored as a positive response to the touch stimulus. Each positive response was followed by touching with a different monofilament that delivered less force, whereas a negative response was followed by a monofilament delivering a greater force. Individual trials were separated by a delay of $30 \mathrm{~s}$ to prevent wind-up, which can result in hyper-excitability of sensory afferents. Twenty trials were performed on each paw to determine the threshold for sensitivity. A paw withdrawal score was calculated as the ratio of the minimum force that evoked withdrawal on the control side to the minimum force that evoked withdrawal on the experimental side; a score of 1.0 indicates equal sensitivity on the two sides. Rats that did not respond to forces less than one that lifted the paw passively ( $180 \mathrm{~g})$ were assigned a score of 0.1 , as indicated by the dashed line in Figure 6C (Lindsey et al., 2000).

\section{Neuroanatomy}

The projections of myelinated sensory axons within the spinal cord were studied by labeling peripheral nerves with cholera toxin B (CTB; SigmaAldrich) injected bilaterally into the radial and/or median nerves of the forelimbs. The nerves were exposed in the forelimb, and $1-2 \mu \mathrm{l}$ of $1-2 \%$ CTB were injected slowly over $10-15$ s into the nerve using a Hamilton syringe with a $26 \mathrm{G}$ needle). Four to seven days later, animals were overdosed with anesthesia and perfused transcardially with $4 \%$ paraformaldehyde. The spinal cord was removed, cryo-protected in 30\% sucrose, and sectioned transversely on a freezing microtome or cryostat. Sections were processed using a polyclonal anti-CTB antibody (List Biologicals; 1:80,000) followed by biotinylated anti-goat secondary antibodies (Vector Laboratories; $1: 200)$. Signal amplification was achieved using avidinbiotin conjugates (ABC kit Vector Laboratories), and antibody binding was visualized using a DAB reaction (DAB kit; Vector Laboratories).

Unmyelinated sensory axons in the spinal cord were identified by processing the sections similarly but using a polyclonal antibody against calcitonin gene-related peptide (CGRP). Cryostat sections were blocked with $20 \%$ fetal bovine serum and incubated with the anti-CGRP antibody (Sigma; 1:4000), followed by the appropriate secondary antibody (Alexa 568; Invitrogen; 1:200). A pixel density analysis was used to measure the extent and distribution of CGRP expression within the dorsal horn (ImageJ software; National Institutes of Health), always comparing the regenerated side with the undamaged contralateral side of the same section. A region of interest was drawn within the dorsal horn surrounding the majority of sensory projections on each side. A threshold pixel intensity was then chosen on the undamaged side and applied equally to both sides. The number of pixels with a density higher than threshold was measured within this region and compared on the two sides. This analysis was performed on three randomly selected sections in the center (C6 or C7) of the deafferented region of spinal cord, providing an anatomical measure of the extent of regeneration. This analysis permitted an assessment of the total extent of regeneration among different animals and also the rostrocaudal distribution of sensory axon regrowth in each animal. MacDermid et al. (2004) used a similar method to estimate the amount of sprouting of unmyelinated sensory axons after dorsal root rhizotomy and $\mathrm{sNgR}$ treatment.

Initially, we were concerned that CTB labeling in rats treated with sNgR might not be specific for myelinated axons. CTB labels medium and large caliber myelinated axons in normal rats but does not label unmyelinated, CGRP ${ }^{+}$axons, as is well documented in the literature and is evident from the absence of label in laminae $\mathrm{I}_{\text {and }} \mathrm{II}_{\mathrm{o}}$ in our own experiments (Figs. $2 \mathrm{~B}, 3 \mathrm{~F}$ ). As described in Results, however, CTBlabeled regenerating axons in rats treated with $\mathrm{sNgR}$ occupy the entire dorsal horn, including the most dorsal laminae where CGRP ${ }^{+}$afferents normally project (Fig. $3 E$ ). It is, therefore, possible that after dorsal root crush and sNgR treatment, both myelinated and unmyelinated sensory axons are labeled with CTB. A similar lack of specificity of CTB labeling for myelinated axons has been reported after injury to peripheral nerves (Tong et al., 1999; Shehab et al., 2003). This possibility is unlikely in the present experiments, however, because $\mathrm{CGRP}^{+}$sensory axons do not regenerate or sprout with $\mathrm{sNgR}$ treatment (Fig. 4). The CTB-labeled sensory axons in laminae $\mathrm{I}_{\text {and }} \mathrm{II}_{\mathrm{o}}$ in $\mathrm{sNgR}$-treated rats are not, therefore, $\mathrm{CGRP}^{+}$, suggesting that CTB does not label unmyelinated sensory afferents even in sNgR-treated rats.

\section{Electrophysiological measurements}

Recordings were made 4-7 d after CTB injections and just before perfusion for histology. Animals were anesthetized using 2.5\% (v/v) isoflurane for the duration of all electrophysiology experiments. The cervical cord was exposed from C4 to T1 and stabilized with a spinal clamp on T2. Radial and/or median nerves were dissected and suspended on silver hooks for stimulation. Recordings were made with metal microelectrodes ( $\sim 200 \mu \mathrm{m}$ exposed tip) positioned $\sim 0.8 \mathrm{~mm}$ lateral to the midline. The radial and median nerves were stimulated separately or together with square $50 \mu \mathrm{s}, 2 \mathrm{~V}$ pulses delivered at $2-5 \mathrm{~Hz}$. Positive voltage changes are shown as upward deflections. Single responses were filtered $(0.1-3 \mathrm{kHz})$, digitized ( 16 bits, $20 \mathrm{kHz}$ sampling rate), averaged (50-100 individual traces), and stored for analysis off line. The spinal preparation usually produced stable, replicable neuronal potentials for several hours.

Recordings were made at each segmental level (C5-T1) on both sides of the cord in response to ipsilateral stimulation of brachial nerves. We recorded first from the experimental (crushed roots) side to ensure that the preparation was in good physiological condition at the time regeneration was assessed. The electrode was advanced vertically into the cord until the synaptic response was maximal, usually at a depth of $0.8-1.0$ $\mathrm{mm}$. The peak magnitude of the field potential, occurring at $2-6 \mathrm{~ms}$ latency, was used as the response amplitude, and the maximum response observed among all recording sites was taken as the global estimate of synaptic function. The fractional recovery of synaptic function, as shown in Figure $5 B$, was defined as the ratio of the maximum response amplitude on the crushed side versus the response amplitude at the corresponding location on the uncrushed side. The time course of the falling phase was determined by comparing the maximum amplitude of the synaptic field potential (typically observed at $2 \mathrm{~ms}$ after stimulus) to the 
response remaining at $6 \mathrm{~ms}$ after stimulus. Return to half maximal response, a more common measure of fall time, was not observable, because the later phases of the response were often contaminated by an EMG response from forelimb muscles. We cut roots that were previously crushed at the end of each experiment to verify that all synaptic responses to peripheral nerve stimulation were abolished.

\section{Results}

\section{Neuroanatomy}

Peripheral nerves of the rat forelimb carry sensory information to the spinal cord via cervical dorsal roots. Two broad classes of sensory axons can be readily distinguished: large diameter myelinated axons that convey information about touch and proprioception and small diameter unmyelinated axons that convey information about temperature and pain. After dorsal root crush, the central projections of sensory axons are interrupted, resulting in degeneration of the axons distal to the injury. These axons regenerate within the dorsal root but stop when they encounter the inhibitory environment of the CNS at the DREZ. To assess whether sensory axons in sNgR-treated rats can cross this barrier and grow back into the spinal cord, we traced the projections of retrogradely labeled sensory axons within the spinal cord.

Normal projections of sensory axons Injection of CTB into the radial and/or median nerves of the forelimb reveals the central projections of transganglionically labeled medium and large diameter sensory afferents in the spinal cord. In normal rats, CTB-labeled sensory afferents were visible within the dorsal columns and in the dorsal gray matter, primarily in laminae III-VI (Fig. $2 B, D, F, H$ ). Labeling was excluded from laminae $\mathrm{I}_{\text {and }} \mathrm{II}_{\mathrm{o}}$ of the dorsal horn (Fig. 2D); these superficial laminae are occupied by small diameter, unmyelinated sensory axons that do not normally transport CTB (LaMotte et al., 1991). In all rats, labeled motoneurons were present in the ventral horn on both the injured and uninjured sides (Fig. $2 A, B)$, which verified that ventral roots were not damaged as a result of the crush surgery and demonstrated that CTB injections into the peripheral nerves were successful even when there was no regeneration of sensory axons. No projections were seen extending from the ventral roots into the dorsal horn, confirming earlier observations by Coggeshall et al. (1980) that myelinated sensory axons do not enter the spinal cord via ventral roots.

Sensory projections after dorsal root crush and no treatment In rats that did not receive $\mathrm{s} \mathrm{NgR}$ treatment, labeled axons in the dorsal roots on the control, uncrushed, side projected through
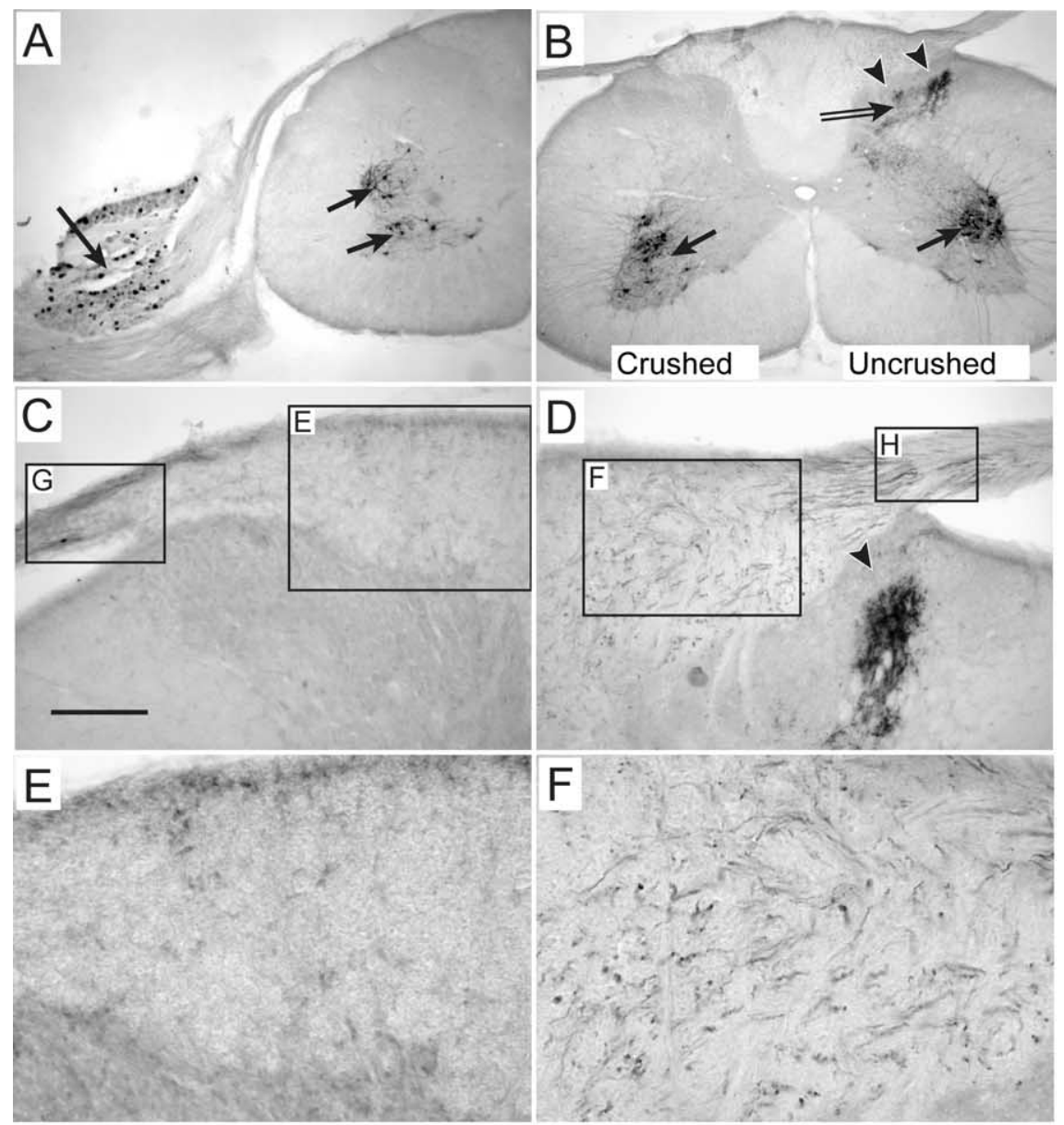

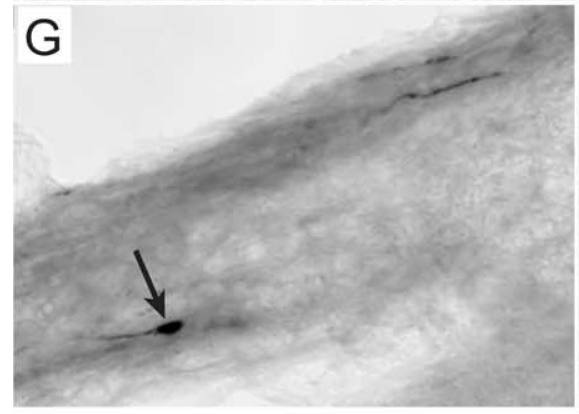

Crushed

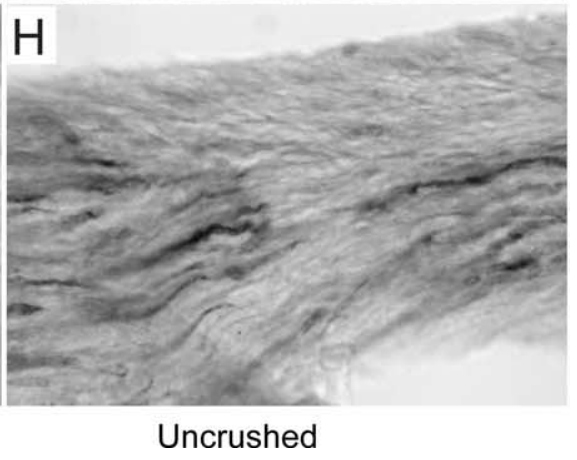

Uncrushed
Figure 2. Spinal projections of myelinated sensory axons in untreated rats. Projections of axons crushed within the dorsal roots 4 weeks previously are shown in $\boldsymbol{A}-\boldsymbol{C}, \boldsymbol{E}$, and $\boldsymbol{G}$; projections of uncrushed axons are shown in $\boldsymbol{B}, \boldsymbol{D}, \boldsymbol{F}$, and $\boldsymbol{H}$. $\boldsymbol{A}, \boldsymbol{B}$, Motoneurons (arrows) and sensory cell bodies (large arrow in $\boldsymbol{A}$ ) are labeled on crushed and uncrushed sides. Projections of sensory axons (double arrow) within the spinal gray matter are only present on the uncrushed side, and these projections avoid the most dorsal spinal laminae in the dorsal horn (arrowheads in $\boldsymbol{B}$ and $\boldsymbol{D}$ ). $\boldsymbol{C}, \boldsymbol{D}$, Labeled axons (shown at higher power in $\boldsymbol{G}$ and $\boldsymbol{H}$ ) are present in dorsal roots on both sides, but after an untreated crush, they end in terminal endbulbs (arrow in $\mathbf{G}$ ) and do not cross into the DREZ. Uncrushed axons project into the dorsal white matter, and many turn to run longitudinally within the dorsal columns (small dots in $\boldsymbol{F}$ ). No labeled axons are seen in the white matter after root crush $(\boldsymbol{E})$. Scale bar in $\boldsymbol{C}$ applies to all panels: $\boldsymbol{A}, \boldsymbol{B}, 500 \mu \mathrm{m} ; \boldsymbol{C}, \boldsymbol{D}, 200$ $\mu \mathrm{m} ; \boldsymbol{E}, \boldsymbol{F}, 100 \mu \mathrm{m} ; \boldsymbol{G}, \boldsymbol{H}, 50 \mu \mathrm{m}$.

the DREZ and into the spinal cord, as in normal rats. The pattern of CTB labeling within the dorsal horn on the uninjured side was normal, and none of the labeled axons projected contralaterally, suggesting that uninjured axons do not sprout in response to contralateral denervation (Fig. $2 A, B$ ). In contrast, axons on the side of the crushed roots stopped at the DREZ and ended in bulb-like structures, as shown in Figure 2, $C$ and $G$. We never observed labeled afferents projecting into the cord from crushed dorsal roots in untreated rats, confirming that crushing the $\mathrm{C} 5-\mathrm{T} 1$ dorsal roots is suf- 

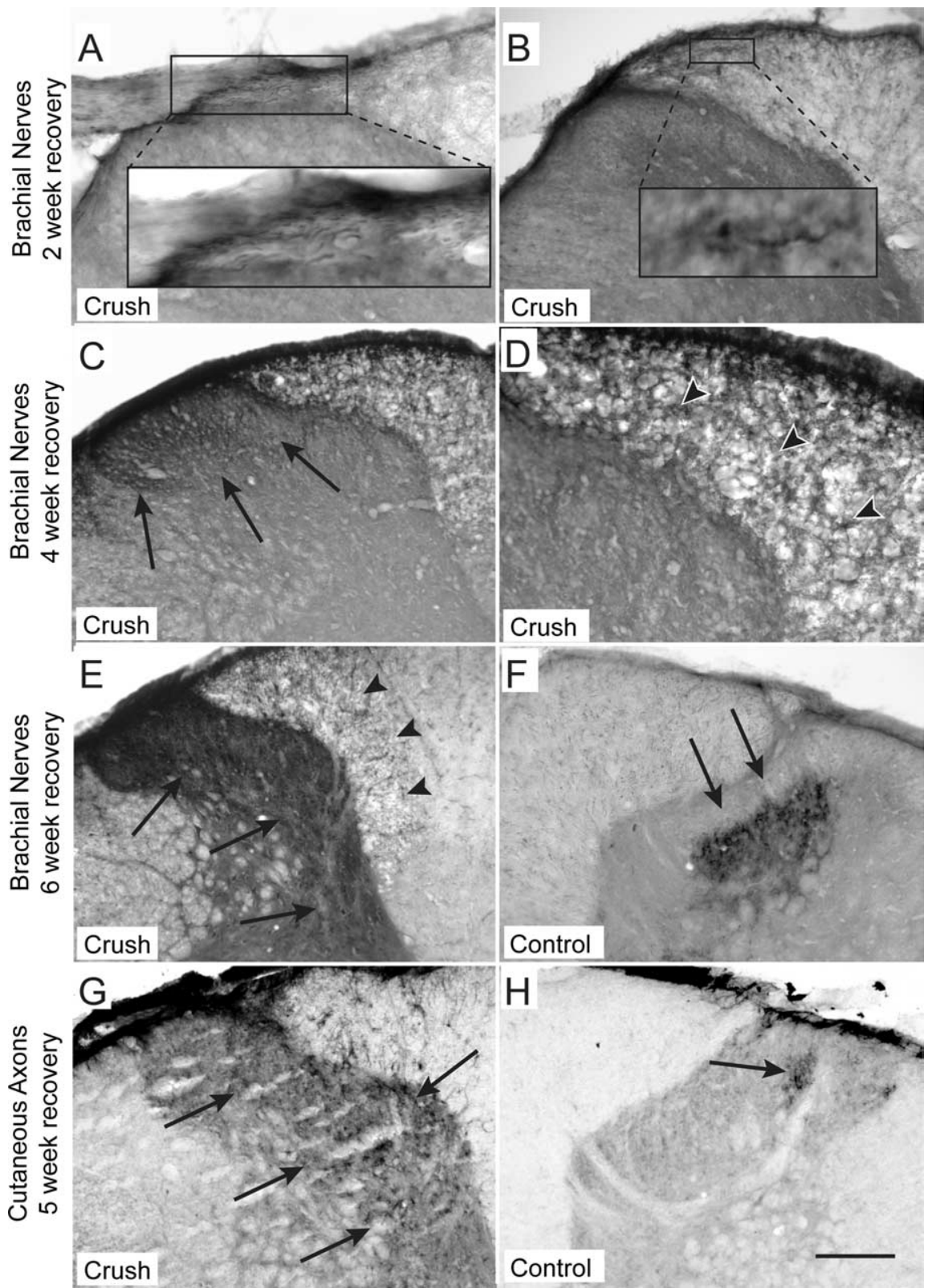

Figure 3. Recovery of spinal projections of lesioned sensory axons in rats treated with $s \mathrm{NgR}$. $A, B$, By 2 weeks, axons labeled with (TB by injection into brachial nerves project into the DREZ $(\boldsymbol{A})$, and occasional axons are seen within the dorsal white matter $(\boldsymbol{B})$. Individual axons are shown at higher magnification in inset panels. $\boldsymbol{C}, \boldsymbol{D}$, By 4 weeks, labeled axons have grown extensively into the dorsal white matter, although they project in a disorganized manner (arrowheads in $\boldsymbol{D}$, which is a higher-magnification view of the white matter shown in $\boldsymbol{C}$. Many project ventrally into the upper laminae of the dorsal horn ( $\boldsymbol{C}$, arrows). $\boldsymbol{E}$, Six weeks after root crush, labeled axons project throughout the dorsal horn (arrows) but still have a disorganized growth pattern in the dorsal white matter (arrowheads). $\boldsymbol{F}$, Uncrushed (TB-labeled axons in a treated rat maintain their normal projection pattern to the dorsal horn, including an absence of projections to laminae I and $\|_{0}(\boldsymbol{F}$, arrows). $\boldsymbol{G}, \boldsymbol{H}$, Regenerated axons supplying a small patch of skin in the arm project diffusely throughout the dorsal horn ( $G$, arrows), unlike the topically restricted projection of uncrushed cutaneous sensory axons on the contralateral side of the same rat (arrow in $\boldsymbol{H}$ ). Scale bars: (in $\boldsymbol{H}) \boldsymbol{A}-\boldsymbol{C}, \boldsymbol{E}-\boldsymbol{H}, 200 \mu \mathrm{m} ; \boldsymbol{D}, 100 \mu \mathrm{m}$.

ficient to interrupt all sensory axons whose peripheral processes run in the radial and median nerves (Fig. 2C,E).

Sensory projections after dorsal root crush and sNgR treatment Treatment with $\mathrm{sNgR}$ led to a gradual recovery of sensory projections to the spinal cord via axons in the crushed dorsal roots. One week after injury, no CTB-labeled axons were visible within the denervated region of the spinal cord in any of the $s \mathrm{NgR}$-treated rats, suggesting that axons were completely interrupted by crushing the roots between the DRG and spinal cord (data not shown).
Labeled cell bodies and axons were present within the DRG and dorsal roots, but the central tips of the axons ended in bulb-like structures at the DREZ, just as seen after root crush in untreated cords. The length of time between injection and killing was sufficient to allow CTB transport between the peripheral nerve and spinal cord, because label was present in motor neurons on both sides of the spinal cord and in the dorsal horn on the uncrushed side (data not shown, but see Figs. $2 B, D, 3 F$ ). At 1 week after crush, no labeled axons were observed in spinal segments outside the region of dorsal root crushes (C5-T1) on either the control or crushed sides, implying that axons in roots rostral and caudal to the crushed roots did not contribute to the labeling pattern observed at longer survival times. Thus, the appearance of labeled axons in denervated segments appearing at later times (see below) is not the result of sprouting of undamaged axons in adjacent roots but rather to growth through the crushed roots.

The first indication of any regrowth of sensory axons appeared at the end of the second postoperative week. CTB-labeled axons had grown through the DREZ and for a short distance into the dorsal white matter, as illustrated in Figure 3, $A$ and $B$. No label was seen elsewhere within the white matter or anywhere in the gray matter. Between the third and fourth weeks after surgery, many labeled axon profiles were visible within the lateral portion of the dorsal white matter, where sensory axons had degenerated (Fig. 3D). Unlike the direct path taken by normal axons, through the DREZ and turning to run longitudinally in the dorsal columns (Fig. $2 D, F)$, CTB labeling in the dorsal columns of treated animals was disorganized, as shown in Figure 3, D and $G$. Labeled sensory axons also projected into the dorsal laminae of the dorsal horn (Fig. 3C). By 6 weeks, many labeled axons projected into spinal laminae III and IV, shown in Figure $3 E$, with a few coursing more ventrally toward the motoneurons. Disorganized axonal projections remained in the lateral portions of the dorsal white matter. These results demonstrate a slow progression of axon growth from the crush site into the dorsal white matter, followed by extension into the gray matter of the dorsal horn. The distribution of axons within the gray matter on the unlesioned sides of all rats, whether treated or untreated, was normal, indicating that $\mathrm{sNgR}$ treatment per se does not disrupt projections of uninjured axons on the side contralateral to the damage (compare Figs. $2 B, D, 3 F, H$ ).

In addition to projections of these axons to their normal target zones, however, CTB-labeled axons also projected densely into laminae $\mathrm{I}$ and $\mathrm{II}_{\mathrm{o}}$, as illustrated in Figure 3, $C$ and $E$. In uninjured 
rats, these laminae are normally occupied by small, unmyelinated sensory axons, which are not labeled by CTB, suggesting that some myelinated regenerating axons project to novel but inappropriate targets in the cord. To examine this possibility more directly, we labeled small groups of cutaneous sensory axons that normally project to a restricted region in the spinal cord. When CTB is injected intradermally into the skin of the shoulder, representing the C6 dermatome, a small population of cutaneous sensory axons is labeled. Sensory axons labeled in this way project to a small region of the dorsal horn, exclusively within the C6 spinal segment, as illustrated in Figure $3 H$. These projections were unaffected by $\mathrm{sNgR}$ treatment when dorsal roots remained intact, providing further evidence that treatment does not cause sprouting of uninjured axons. In contrast to this restricted projection, however, the spinal projection of regenerated sensory axons labeled in the same manner was widespread. As illustrated in Figure $3 G, 5$ weeks after dorsal root crush with $\mathrm{sNgR}$ treatment, regenerated cutaneous axons projected diffusely within the dorsal white matter, similar to that observed with whole-nerve injections, and the limited projections into the dorsal horn were not restricted to a small region. Labeled cutaneous axons also projected within the dorsal columns several segments both rostral and caudal to the C6 segment, unlike the segmentally restricted projections of unlesioned axons with or without $\mathrm{sNgR}$ (data not shown). These results demonstrate that at least some classes of sensory axons regenerate into inappropriate regions of the spinal cord.

Nociceptive sensory axons do not regenerate with $s \mathrm{NgR}$ treatment A possible explanation for the aberrant projection of CTBlabeled myelinated axons into laminae $\mathrm{I}_{\text {and }} \mathrm{II}_{\mathrm{o}}$ is that the unmyelinated sensory axons, which normally occupy these laminae, fail to regenerate in sNgR-treated animals. In the absence of the normal sensory innervation of these laminae, myelinated afferents might regenerate aberrantly into this region. Many of the unmyelinated C-fibers normally projecting to these dorsal laminae express CGRP, a neuropeptide that is expressed by nociceptive sensory axons, as illustrated in Figure $4 \mathrm{~B}$. We, therefore, used CGRP expression to assess the extent and distribution of nociceptive axonal projections after dorsal root crush (Lawson, 1995; Bennett et al., 1996).

In untreated rats 4 weeks after cervical dorsal root crushes, most CGRP expression disappeared in segments located within the crush region (for example, the $\mathrm{C} 7$ segment with crushes from C5 to T1), as shown in Figure 4A. Measurements of the density of CGRP labeling in the dorsal horn revealed that CRGP expression fell to $15 \pm 7 \%$ ( $n=5$ rats $)$ of the normal, contralateral side. Most normal CGRP expression within the C7 segment is, therefore, from sensory axons in the C5-T1 dorsal roots, and relatively little is contributed by sensory axons from flanking, uncrushed roots. The CGRP ${ }^{+}$axons that did persist on the crushed side, presumably from unmyelinated afferents in adjacent, uninjured segments, were similarly restricted to laminae $\mathrm{I}$ and $\mathrm{II}_{\mathrm{o}}$, suggesting
Crushed Uncrushed

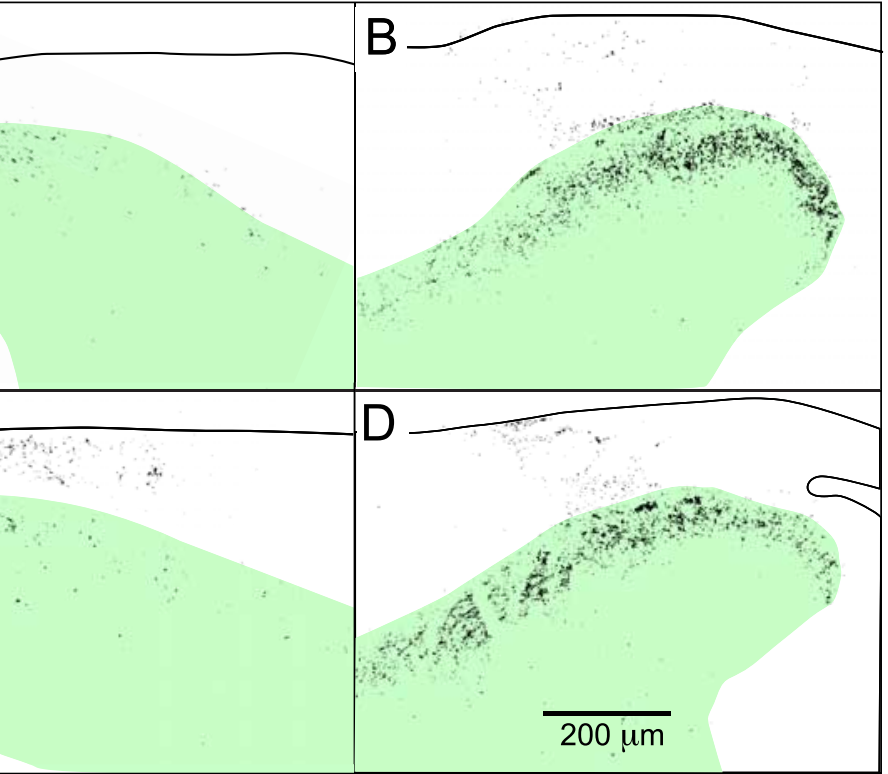

Figure 4. Distribution and density of CGRP expression in treated and untreated rats 4 weeks after unilateral root crush. Each panels is from the two sides of the same transverse section. Pixel density of fluorescently labeled sections was thresholded on schematic drawings of the section. The dorsal horn is shown in green. There is little recovery of CGRP expression after root crush

that spared axons within the denervated region of untreated cords do not sprout into novel regions.

At 4 weeks after lesion with sNgR-treatment, CGRP expression on the crushed side was unchanged in the denervated region, to $17 \pm 2 \%$ ( $n=17$ rats) of normal, with a distribution similar to that in unlesioned animals, illustrated in Figure 4, $C$ and $D$. The absence of an increase in CGRP expression suggests that $\mathrm{sNgR}$ does not promote regeneration of this class of sensory fibers, nor does it induce sprouting of uninjured fibers in adjacent segments, whose dorsal roots were spared. The absence of significant sprouting is in agreement with earlier findings demonstrating that the only increases in CGRP expression after sNgR treatment after dorsal root lesions are small and limited to deeper laminae (MacDermid et al., 2004). In contrast, delivery of NGF or FGF to the spinal cord after dorsal root crush promotes robust regeneration and sprouting of CGRP ${ }^{+}$axons (Romero et al., 2001). Unmyelinated sensory axons, therefore, have the ability to regenerate and sprout in the spinal cord, but $s \mathrm{NgR}$ is not an appropriate stimulus for growth.

\section{Electrophysiology}

We were interested in determining whether the extensive regeneration observed anatomically also resulted in the formation of functional synaptic connections within the spinal cord. Electrophysiological recording provides a quantitative method for assessing the extent to which regenerated axons reestablish functional contacts with spinal neurons. Field potentials evoked by stimulation of the median and ulnar forelimb nerves were recorded extracellularly in the spinal gray matter. In normal rats, evoked responses are composed of two separate peaks (Fig. 5A). The first peak represents synchronized action potentials in the DRG and/or dorsal roots, because it persists after cutting dorsal roots. The second, broader peak, with a latency of $\sim 1.5 \mathrm{~ms}$ after the stimulus, represents the postsynaptic responses evoked by activity in low-threshold sensory afferents in forelimb nerves. 
A

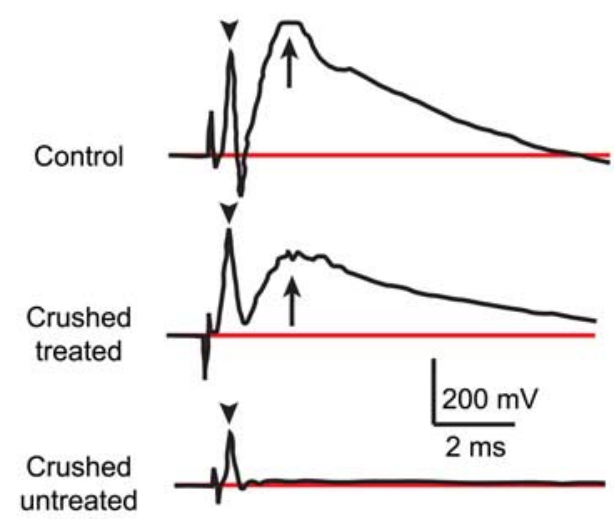

B

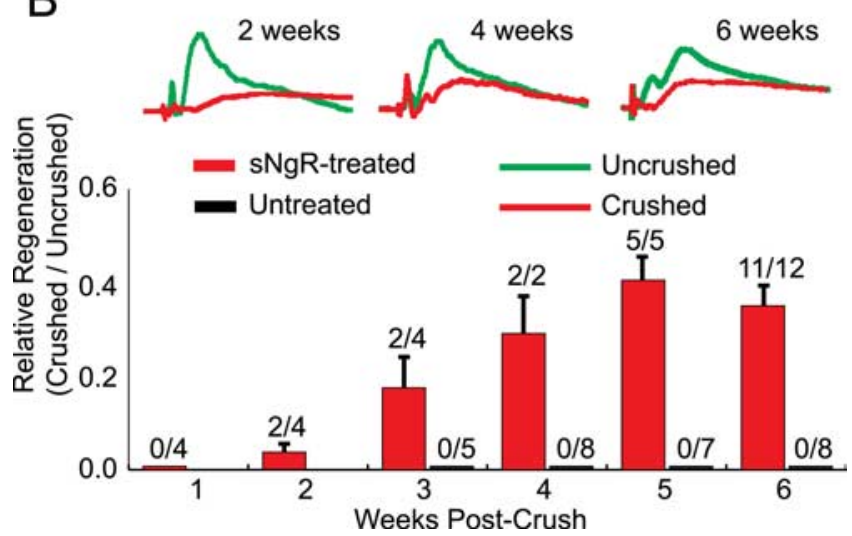

Figure 5. Functional recovery of synaptic activity evoked by peripheral nerve stimulation in treated spinal cords. A, Examples of extracellular field potentials recorded in the spinal cord after stimulation of the median and ulnar nerves. The first peak after the stimulus (arrowheads) represents synchronized action potentials in the DRG and dorsal roots. The second peak (arrows) represents postsynaptic responses in spinal neurons. $\boldsymbol{B}$, Time course of recovery of synaptic function after root crush. Representative traces at 2, 4, and 6 weeks are shown in the top; red traces are evoked by sensory axons that were previously crushed, green traces from the contralateral, control side of the same rat. Bars indicate fractional recovery of synaptic potential amplitudes. Error bars indicate \pm 1 SEM. The numbers above each column indicate the number of rats with measurable synaptic responses versus the total number of rats tested at each time point.

The peak amplitudes of these synaptic field potentials, which occurred $\sim 3 \mathrm{~ms}$ after the stimulus, were $250-1500 \mu \mathrm{V}$.

When dorsal roots corresponding to those that are crushed are cut acutely in uninjured animals, the field potentials within the central segments (C6-C8) of the deafferented cord disappear. These responses are, therefore, mediated by sensory axons in the C5-T1 dorsal roots, and they cannot be accounted for by afferents entering the spinal cord either through ventral roots or through other roots in adjacent, uninjured segments. Even 6 weeks after root crush, animals without treatment exhibited no synaptic responses in the deafferented region of the spinal cord, as illustrated in Figure 5, consistent with the absence of CTB-labeled projections in this region. There was no significant regeneration of sensory fibers in any of the 28 untreated rats.

With $\mathrm{sNgR}$ treatment, there was a slow but reproducible recovery of synaptic function, as illustrated in Figure $5 B$. No electrophysiological responses were observed 1 week after injury, consistent with a lack of anatomical projections in the denervated region. At the end of the second week, there were small responses in two of four rats, but the average response for all four rats did not reach significance. By 3 weeks, however, the average response for all four animals tested was significantly larger than zero, although only two of these animals had clear responses. Both animals with positive functional responses showed neuroanatomical evidence of axon growth past the dorsal root entry zone and into the spinal cord. In contrast, the one other animal at this time point analyzed anatomically had labeled axons in the DREZ but not further into the spinal cord. We saw no delay between anatomical regeneration and the return of physiological responses.

At recovery times of $>3$ weeks, 18 of the $19 \mathrm{sNgR}$-treated rats had significant synaptic responses, as illustrated in Figure $5 B$. Amplitudes of these responses were $\sim 40 \%$ of the unlesioned side (36 $\pm 5 \%, p<0.001$, including all 19 animals). There were several differences between the responses on the regenerated and control sides of individual animals. The average latency to peak amplitude of the regenerated response was 3.9 ms compared with $2.3 \mathrm{~ms}$ on the control side, a significant increase $(p<0.001)$. This increase is likely the result of the smaller diameter and/or incomplete remyelination of the regenerated sensory axons. The falling phase of regenerated responses was also slower than normal. At 6 $\mathrm{ms}$ after the stimulus, normal synaptic responses had fallen to $44 \pm 3 \%$ of their peak values, whereas regenerated responses were still at $76 \pm 3 \%$ of their peak values, a significant difference $(p<$ 0.001 , see Materials and Methods for why fall times were measured in this manner). A probable explanation for the slower time course of regenerated responses is that there is a larger contribution from polysynaptic connections. A normal range of response amplitudes on the unlesioned side was observed with latencies and falling phases that were similar to responses in unlesioned, untreated animals, suggesting that the connections of uninjured axons are not affected by sNgR treatment.

\section{Behavior}

Anatomical and electrophysiological tests indicated extensive regeneration of lesioned sensory axons in treated animals. We, therefore, assessed if this regeneration led to a corresponding improvement in the ability of these animals to use their affected forelimb. Behavioral improvements in limb use were monitored twice weekly throughout the survival period. Three tests were used to evaluate functional recovery of the limb: asymmetry of forelimb use, grasping, and sensitivity to touch.

The forelimb asymmetry test was used to evaluate general use of the limb (Schallert et al., 2000). When placed in a glass cylinder, rats explore the wall by placing their forepaws on the glass. In normal rats, the two forepaws of uninjured animals are used equally, but after brachial dorsal root lesions, the affected forepaw is not used. As shown in Figure 6A, none of the rats with unilateral C5-T1 dorsal root crushes but without $\mathrm{sNgR}$ treatment used their affected forelimb to explore the inside of the cylinder. The compromised limb remained straight at the side of the animal when rearing, often with a flexed wrist. This deficit persisted throughout the survival period.

Rats that had crushed roots and were treated with sNgR, however, did progressively recover partial use of their forelimbs, as shown in Figure 6A. No improvement in limb use was seen after 1 week, consistent with the absence of anatomical or functional synaptic recovery at this time. After the second week, however, sNgR-treated rats used their denervated limb $\sim 10 \%$ as often as the normal limb, corresponding to the small (and nonsignificant) recovery of synaptic function at this time. By 3 weeks, forelimb use increased to $\sim 20 \%$ of use on the normal side, but the use was still quite variable, consistent with the improvement but contin- 

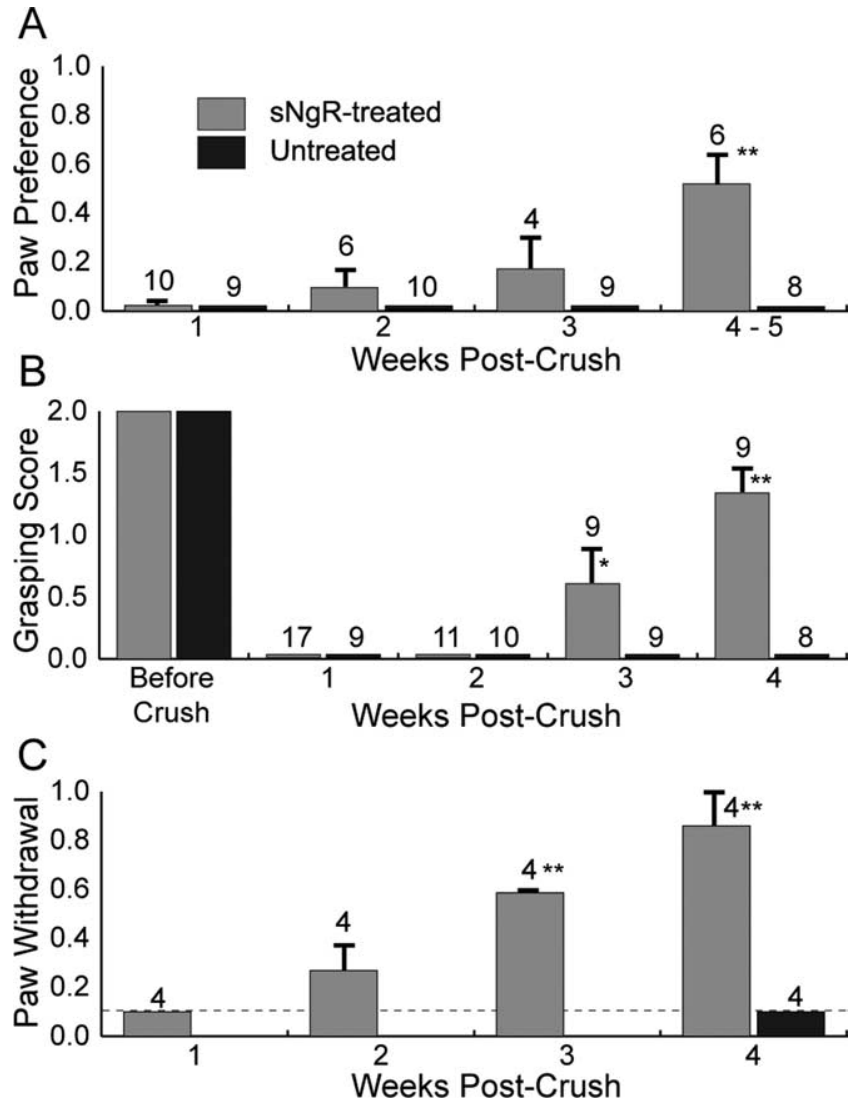

Figure 6. Behavioral recovery of $\mathrm{s} \mathrm{NgR}$-treated and untreated rats after root crush. The number of rats tested at each time point is indicated above each bar. The legend applies to all panels. Asterisks indicate significant differences from treated rats at 1 week: ${ }^{*} p<0.01$; ${ }^{* *} p<$ 0.001 . Error bars indicate \pm 1 SEM. $\boldsymbol{A}$, Paw preference test. $\boldsymbol{B}$, Ability to grasp. $\boldsymbol{C}$, Withdrawal of forelimb to tactile stimulation of paw. The dotted line in $C$ represents the minimum sensitivity this test could measure (see Materials and Methods). Paw withdrawal was only measured at 4 weeks for untreated rats.

ued variability in synaptic function. At 4 weeks after lesion, rats exhibited $\sim 50 \%$ of their normal forelimb use, and this function did not increase over the following 2 weeks. Even when forelimb use improved, however, normal use of the limb was not restored. The affected forelimb was rarely used to support weight while standing, and the dorsal surface of the paw was often in contact with the floor of the cage.

Grasping requires that a rat be able to sense when its paw touches a bar and then react by flexing its toes. The test of grasping ability was used to evaluate how the forepaw responded to tactile stimuli. Rats were suspended above a metal bar by the tail and lowered so the forepaws were within reach of the bar. Normal animals grasp the bar strongly with both paws and resist being withdrawn from it. As shown in Figure $6 B$, none of the untreated rats could position its limb properly, and none was able to grasp (see rating scale in Materials and Methods). The affected forelimb remained straight and close to the body, and only limited, undirected movement of the limb was observed. The ability of the normal, contralateral forepaw to grasp remained normal.

Treatment with $\mathrm{sNgR}$ also resulted in a delayed but significant improvement in grasping. As shown in Figure $6 \mathrm{~B}$, recovery did not begin until the third postoperative week, and at that time, some rats still showed no improvement, although the recovery at this time was significant $(p<0.01)$. At the end of 4 weeks, all rats with significant recovery of synaptic re- sponses were able to direct the movement of their forepaw toward the bar and grasp (grasping score of $1.3 \pm 0.9, p<$ $0.001)$. The strength of grasping was qualitatively less than that observed on the unlesioned side, but animals were able to resist moderate withdrawal force. The one rat that did not exhibit synaptic recovery as assessed electrophysiologically (1 of 19 rats) also did not recover the ability to grasp.

The third behavioral test provided an assessment of the threshold for paw withdrawal in response to tactile stimulation. Measurement of the force, provided by a von Frey hair, required to elicit a withdrawal response not only tests if the animal can sense and respond to a touch but also if normal touch stimuli evoke nociceptive responses. The development of allodynia is of particular concern when developing treatments for CNS injuries, because sprouting or regeneration may promote pain syndromes. We, therefore, sought to determine if $s \mathrm{NgR}$-treated rats with root injuries develop normal sensation in the affected forepaw and if the range of stimuli that evoke responses on the unaffected side remains normal. Both forepaws of uninjured animals are sensitive to approximately equal forces, giving a paw withdrawal score of 1.0 (data not shown). With unilateral dorsal root crush, the forepaw ipsilateral to injury became insensitive to any force less than that required to passively lift the paw (Fig. 6C, dashed line).

Tactile sensation of the forepaw, as measured by withdrawal from tactile stimulation, began to recover in rats treated with $\mathrm{sNgR}$ at the end of the second postoperative week (27\%, not significant) and reached $59 \%$ of the normal side by the end of 3 weeks $(p<0.001)$, as shown in Figure $6 C$. Sensitivity gradually returned to near-normal levels at the end of 4 weeks $(86 \%, p<$ $0.001)$. All treated animals responded to a normal range of stimuli, and none of the stimuli evoked responses that appeared to be painful, indicating that $\mathrm{sNgR}$ treatment does not induce allodynia. It is unclear why recovery of tactile input occurred earlier than other measures of regeneration, although a similar time course of recovery was seen with artemin treatment in an earlier study (Wang et al., 2008). Animals with dorsal root crushes that did not receive $\mathrm{sNgR}$ did not exhibit any behavioral recovery, as tested explicitly at the end of the recovery period. Responses measured on the contralateral side remained within the normal range, in agreement with neuroanatomical data indicating that connections on the uninjured side remain undisturbed by $\mathrm{sNgR}$ treatment.

\section{Discussion}

Blocking the inhibitory influence of $\mathrm{NgR}$ ligands with $\mathrm{sNgR}$ promoted the regeneration of myelinated sensory axons through the DREZ and into the white and gray matter of the spinal cord. Unmyelinated sensory afferents did not regenerate, however. Growth of myelinated afferents was observed beginning at the end of the second postoperative week, and by the third week, growing axons extended into the gray matter. In concert with the anatomical growth of sensory axons into the cord, there was also a gradual restoration of synaptic function in the denervated region, as revealed by microelectrode recordings from the spinal gray matter in response to stimulation of peripheral nerves. These positive synaptic responses were correlated with improved behavior. Importantly, sNgR treatment did not modify the anatomical projections of sensory axons on the contralateral side where dorsal roots remained intact, including the specific projections of cutaneous axons to topographically restricted regions of the dorsal horn. These results suggest that $\mathrm{sNgR}$ can promote the func- 
tional regeneration of lesioned sensory axons without disrupting the normal projections of uninjured axons.

In this study, the inhibitory effects of myelin-associated proteins on axon growth were abrogated using $\mathrm{sNgR}$, a soluble peptide of the binding site on the Nogo-66 receptor, NgR1, coupled to an antibody fragment. Nogo, OMgp, and MAG all bind the leucine-rich repeat region of NgR1 (Barton et al., 2003), so sNgR, therefore, binds to all three proteins. The sequestration of NgR1 ligands with $s \mathrm{NgR}$ not only blocks their interaction with $\mathrm{NgR} 1$ ( $\mathrm{Li}$ et al., 2004) but also may interfere with binding to other, unknown receptors. The potent growth-promoting effects of $s \mathrm{NgR}$ on regenerating sensory axons may result from this generalized blockade, especially in light of the recent identification of PirB as another receptor mediating the inhibitory effects of myelin (Atwal et al., 2008). Before the present report, the only published accounts of the effects of sNgR in vivo were on sprouting from spared axons (MacDermid et al., 2004; Ji et al., 2005; Li et al., 2005; Wang et al., 2006), so it will be important to determine if this peptide also promotes regeneration of other classes of spinal axons.

Another factor that contributes to inhibition of sensory axon growth through the DREZ is the expression of chondroitin sulfate proteoglycans, which are upregulated in the DREZ after dorsal root crush (Steinmetz et al., 2005). Digestion of these CSPGs with chondroitinase $\mathrm{ABC}(\mathrm{ChABC})$, when combined with a growth-promoting pretreatment of sensory neurons with zymosan, leads to anatomical and functional regeneration of axons into the spinal cord (Steinmetz et al., 2005). Treatment with ChABC also promotes sprouting of spared sensory axons after rhizotomy of several adjacent dorsal roots (Cafferty et al., 2008). A combined therapy of growth stimulation (Wang et al., 2008) and blockade of multiple inhibitory factors, therefore, may prove useful for achieving maximal recovery from root injuries.

\section{Can recovery of function be explained by sprouting from spared axons?}

We used the dorsal root crush paradigm to study axonal regeneration within the inhibitory environment of the adult CNS. Several features of this preparation facilitate the ability to discriminate between recovery based on true regeneration from that based on sprouting from axons that enter the spinal cord via other pathways that are not damaged by the crush. First, virtually all myelinated sensory axons enter the spinal cord via dorsal roots, so lesions of these roots eliminate all segmental inputs to the lesioned segments. Acute dorsal root lesions from C5 to T1 abolish all sensory projections from the median, radial and ulnar nerves, based both on CTB labeling and electrical stimulation of these nerves. In sNgR-treated rats in which sensory function has recovered, cutting the dorsal roots that had previously been crushed always abolished the electrical responses. These results demonstrate that all the functional recovery of sensory inputs to the cord is mediated by axons entering the cord via previously crushed dorsal roots. The reduction of CGRP expression by $>80 \%$ within brachial segments after C5-T1 root crushes with or without $\mathrm{sNgR}$-treatment indicates that the majority of unmyelinated axonal projections to these segments are also interrupted by these lesions.

Another potential source of spared axons stems from the possibility that crush lesions may not interrupt all axons in the dorsal root. The recovery reported here might then result from sprouting of undamaged, or "spared," axons in the crushed roots (Steward et al., 2003). Our technique of root crush is identical to the one we used in an earlier study (Wang et al., 2008). In that study, we demonstrated that retrograde tracing from the spinal cord with CTB $2 \mathrm{~d}$ after root crush labels virtually no sensory neurons in the DRG (Fig. 7) [see Wang et al. (2008), their Fig. S6]. It is, therefore, unlikely that our crush technique leaves a sufficient number of myelinated sensory axons that survive the crush to sprout and restore $40 \%$ of normal synaptic input to spinal neurons.

The progressive invasion of the spinal cord by labeled sensory projections and its correlation with functional recovery provides further strong support for the use of the dorsal root crush paradigm as an appropriate model in which to evaluate the efficacy of agents to promote regeneration within the spinal cord. Early restoration of function within 1 week of lesion would strongly implicate an incomplete lesion, but the time course of recovery we observed was slower and is in accord with estimates for regeneration-based recovery suggested by others (Steward et al., 2003). Labeled axons projecting into the spinal cord are not visible until the end of the second week. This delayed growth also correlates temporally with the appearance of small but measurable synaptic responses in the denervated region of the cord. A progressive increase in axon growth was observed both anatomically and functionally at weekly intervals after lesion. The anatomical and electrophysiological studies, therefore, provide strong evidence that virtually all sensory axons, especially myelinated axons that mediate CTB transport and the short latency electrical responses, are lesioned after root crush.

The ectopic location of many of the CTB-labeled axons in treated animals also supports the interpretation that recovery is mediated by regenerating axons. The disorganized nature of labeled axons within the dorsal columns is in contrast to the normal longitudinal projections of these axons in the dorsal white matter. Labeled axons after recovery from root crush also occupy the most dorsal laminae of the dorsal horn, whereas CTB-labeled axons do not project to laminae I and $\mathrm{II}_{\mathrm{o}}$ in normal rats. Similarly, axons labeled with intradermal injections of CTB into the upper forelimb do not project to their normal target zones. Instead, these axons project aberrantly in the white matter and weakly throughout the dorsal gray matter.

The possibility that small diameter unmyelinated axons escape damage after root crush was also assessed. Many unmyelinated sensory axons express CGRP, which is, therefore, a useful marker for their presence. One week after dorsal root crush, both treated and untreated rats had only a low level of CGRP expression on the lesioned side, suggesting that few if any CGRP ${ }^{+}$axons were spared after dorsal root crushes. The absence of increased expression of CGRP in the cord even several weeks after $\mathrm{sNgR}$ treatment further supports our conclusion that these axons do not regenerate, nor do they sprout from adjacent segments. Weak or negligible sprouting by $\mathrm{CGRP}^{+}$sensory axons has also been reported by MacDermid et al. (2004) in rats subjected to avulsion of multiple dorsal roots followed by treatment with sNgR.

\section{Do regenerated axons restore connections to supraspinal areas?}

In the dorsal root crush model, regenerated axons are required to grow only short distances to make contacts with targets in the spinal cord. Work by other investigators has suggested that the ability to grasp may involve projections to supraspinal areas. Although we cannot address this issue directly, our impression is that the recovery of grasping ability promoted by sNgR treatment is too rapid to be accounted for by long-distance axonal growth. 
A plausible explanation of this apparent contradiction is that the grasping test used here does not require sensory input to supraspinal levels. Unilateral lesions of the medial half of the spinal cord at C5, which abolishes ascending sensory inputs, lead to permanent loss of the grasping reflex (Anderson et al., 2007). These lesions also interrupt the main corticospinal tract in the dorsal columns, however, so the loss of grasping may be caused by loss of descending input to the cord. A more direct test of the role of ascending sensory input on forepaw grasping was made using lesions at C2 restricted to the dorsal portions of the dorsal columns, thereby lesioning rostrally projecting sensory axons but sparing most of the corticospinal tract (Ballermann et al., 2001). These lesions disrupted the ability to make fine tactile discriminations, but did not interfere with simple grasping. The simple grasping test used in the present experiments, therefore, probably did not require long-distance regeneration of sensory fibers to higher centers. The restricted lesions used by Ballermann et al. (2001) also did not disrupt limb use as assessed by the forelimb asymmetry test, indicating that these behaviors also do not require sensory projections to higher centers.

\section{Does treatment with $\mathrm{sNgR}$ induce neuropathic pain?}

Expansion of the projections of myelinated afferents into the superficial laminae of the spinal cord has been implicated in the development of pain syndromes (Woolf et al., 1992; Lekan et al., 1996; Mannion et al., 1996). In the dorsal root crush model, sNgR promoted aberrant growth of myelinated axons into laminae normally occupied by nociceptive axons. Although CGRP ${ }^{+} \mathrm{ax}^{-}$ ons apparently neither regenerate nor sprout from adjacent segments with sNgR treatment, we were concerned that the abnormal projections of myelinated fibers might result in allodynia. Regeneration did result in restoration of tactile sensation in the forepaw, but there was no evidence that rats became hypersensitive to these stimuli. Allodynia is, therefore, unlikely to be a complication for $\mathrm{sNgR}$ therapy. A similar absence of allodynia was noted in an earlier study using a monoclonal antibody against NogoA to treat spinal cord lesions (Merkler et al., 2001).

\section{References}

Anderson KD, Gunawan A, Steward O (2007) Spinal pathways involved in the control of forelimb motor function in rats. Exp Neurol 206:318-331.

Atwal JK, Pinkston-Gosse J, Syken J, Stawicki S, Wu Y, Shatz C, TessierLavigne M (2008) PirB is a functional receptor for myelin inhibitors of axonal regeneration. Science 322:967-970.

Ballermann M, McKenna J, Whishaw IQ (2001) A grasp-related deficit in tactile discrimination following dorsal column lesion in the rat. Brain Res Bull 54:237-242.

Barton WA, Liu BP, Tzvetkova D, Jeffrey PD, Fournier AE, Sah D, Cate R, Strittmatter SM, Nikolov DB (2003) Structure and axon outgrowth inhibitor binding of the Nogo-66 receptor and related proteins. EMBO J 22:3291-3302.

Bennett DL, Dmietrieva N, Priestley JV, Clary D, McMahon SB (1996) trkA, CGRP and IB4 expression in retrogradely labelled cutaneous and visceral primary sensory neurones in the rat. Neurosci Lett 206:33-36.

Cafferty WB, Bradbury EJ, Lidierth M, Jones M, Duffy PJ, Pezet S, McMahon SB (2008) Chondroitinase ABC-mediated plasticity of spinal sensory function. J Neurosci 28:11998-12009.

Carlstedt T (1997) Nerve fibre regeneration across the peripheral-central transitional zone. J Anat 190:51-56.

Chivatakarn O, Kaneko S, He Z, Tessier-Lavigne M, Giger RJ (2007) The Nogo-66 receptor NgR1 is required only for the acute growth conecollapsing but not the chronic growth-inhibitory actions of myelin inhibitors. J Neurosci 27:7117-7124.

Coggeshall RE, Maynard CW, Langford LA (1980) Unmyelinated sensory and preganglionic fibers in rat L6 and S1 ventral spinal roots. J Comp Neurol 193:41-47.

Fawcett JW, Rokos J, Bakst I (1989) Oligodendrocytes repel axons and cause axonal growth cone collapse. J Cell Sci 92:93-100.

Fournier AE, Gould GC, Liu BP, Strittmatter SM (2002) Truncated soluble Nogo receptor binds Nogo-66 and blocks inhibition of axon growth by myelin. J Neurosci 22:8876-8883.

Goldberg JL, Barres BA (2000) The relationship between neuronal survival and regeneration. Annu Rev Neurosci 23:579-612.

Ji B, Li M, Budel S, Pepinsky RB, Walus L, Engber TM, Strittmatter SM, Relton JK (2005) Effect of combined treatment with methylprednisolone and soluble Nogo-66 receptor after rat spinal cord injury. Eur J Neurosci 22:587-594.

Kim JE, Liu BP, Park JH, Strittmatter SM (2004) Nogo-66 receptor prevents raphespinal and rubrospinal axon regeneration and limits functional recovery from spinal cord injury. Neuron 44:439-451.

LaMotte CC, Kapadia SE, Shapiro CM (1991) Central projections of the sciatic, saphenous, median, and ulnar nerves of the rat demonstrated by transganglionic transport of choleragenoid-HRP (B-HRP) and wheat germ agglutinin-HRP (WGA-HRP). J Comp Neurol 311:546-562.

Lawson SN (1995) Neuropeptides in morphologically and functionally identified primary afferent neurons in dorsal root ganglia: substance $\mathrm{P}$, CGRP and somatostatin. Prog Brain Res 104:161-173.

Lekan HA, Carlton SM, Coggeshall RE (1996) Sprouting of A beta fibers into lamina II of the rat dorsal horn in peripheral neuropathy. Neurosci Lett 208:147-150.

Li S, Liu BP, Budel S, Li M, Ji B, Walus L, Li W, Jirik A, Rabacchi S, Choi E, Worley D, Sah DW, Pepinsky B, Lee D, Relton J, Strittmatter SM (2004) Blockade of Nogo-66, myelin-associated glycoprotein, and oligodendrocyte myelin glycoprotein by soluble Nogo-66 receptor promotes axonal sprouting and recovery after spinal injury. J Neurosci 24:10511-10520.

Li S, Kim JE, Budel S, Hampton TG, Strittmatter SM (2005) Transgenic inhibition of Nogo-66 receptor function allows axonal sprouting and improved locomotion after spinal injury. Mol Cell Neurosci 29:26-39.

Lindsey AE, LoVerso RL, Tovar CA, Hill CE, Beattie MS, Bresnahan JC (2000) An analysis of changes in sensory thresholds to mild tactile and cold stimuli after experimental spinal cord injury in the rat. Neurorehabil Neural Repair 14:287-300.

MacDermid VE, McPhail LT, Tsang B, Rosenthal A, Davies A, Ramer MS (2004) A soluble Nogo receptor differentially affects plasticity of spinally projecting axons. Eur J Neurosci 20:2567-2579.

Mannion RJ, Doubell TP, Coggeshall RE, Woolf CJ (1996) Collateral sprouting of uninjured primary afferent A-fibers into the superficial dorsal horn of the adult rat spinal cord after topical capsaicin treatment to the sciatic nerve. J Neurosci 16:5189-5195.

Merkler D, Metz GA, Raineteau O, Dietz V, Schwab ME, Fouad K (2001) Locomotor recovery in spinal cord-injured rats treated with an antibody neutralizing the myelin-associated neurite growth inhibitor Nogo-A. J Neurosci 21:3665-3673.

Ramer MS, Priestley JV, McMahon SB (2000) Functional regeneration of sensory axons into the adult spinal cord. Nature 403:312-316.

Ramon y Cajal S (1928) Degeneration and regeneration of the nervous system. London: Oxford UP.

Romero MI, Rangappa N, Garry MG, Smith GM (2001) Functional regeneration of chronically injured sensory afferents into adult spinal cord after neurotrophin gene therapy. J Neurosci 21:8408-8416.

Schallert T, Fleming SM, Leasure JL, Tillerson JL, Bland ST (2000) CNS plasticity and assessment of forelimb sensorimotor outcome in unilateral rat models of stroke, cortical ablation, parkinsonism and spinal cord injury. Neuropharmacology 39:777-787.

Schwab ME (2004) Nogo and axon regeneration. Curr Opin Neurobiol 14:118-124.

Shehab SA, Spike RC, Todd AJ (2003) Evidence against cholera toxin B subunit as a reliable tracer for sprouting of primary afferents following peripheral nerve injury. Brain Res 964:218-227.

Steinmetz MP, Horn KP, Tom VJ, Miller JH, Busch SA, Nair D, Silver DJ, Silver J (2005) Chronic enhancement of the intrinsic growth capacity of sensory neurons combined with the degradation of inhibitory proteoglycans allows functional regeneration of sensory axons through 
the dorsal root entry zone in the mammalian spinal cord. J Neurosci 25:8066-8076.

Steward O, Zheng B, Tessier-Lavigne M (2003) False resurrections: distinguishing regenerated from spared axons in the injured central nervous system. J Comp Neurol 459:1-8.

Tong YG, Wang HF, Ju G, Grant G, Hökfelt T, Zhang X (1999) Increased uptake and transport of cholera toxin B-subunit in dorsal root ganglion neurons after peripheral axotomy: possible implications for sensory sprouting. J Comp Neurol 404:143-158.

Venkatesh K, Chivatakarn O, Lee H, Joshi PS, Kantor DB, Newman BA, Mage R, Rader C, Giger RJ (2005) The Nogo-66 receptor homolog NgR2 is a sialic acid-dependent receptor selective for myelin-associated glycoprotein. J Neurosci 25:808-822.

Wang R, King T, Ossipov MH, Rossomando AJ, Vanderah TW, Harvey P,
Cariani P, Frank E, Sah DW, Porreca F (2008) Persistent restoration of sensory function by immediate or delayed systemic artemin after dorsal root injury. Nat Neurosci 11:488-496.

Wang X, Baughman KW, Basso DM, Strittmatter SM (2006) Delayed Nogo receptor therapy improves recovery from spinal cord contusion. Ann Neurol 60:540-549.

Woolf CJ, Shortland P, Coggeshall RE (1992) Peripheral nerve injury triggers central sprouting of myelinated afferents. Nature 355:75-78.

Yiu G, He Z (2006) Glial inhibition of CNS axon regeneration. Nat Rev Neurosci 7:617-627.

Zheng B, Atwal J, Ho C, Case L, He XL, Garcia KC, Steward O, TessierLavigne M (2005) Genetic deletion of the Nogo receptor does not reduce neurite inhibition in vitro or promote corticospinal tract regeneration in vivo. Proc Natl Acad Sci U S A 102:1205-1210. 